\title{
Effects of Lower Body Positive Pressure on Cardiovascular Responses During Walking in Elderly Women
}

\author{
T. SOTA ${ }^{1}$, S. MATSUO ${ }^{2}$, Y. UCHIDA ${ }^{3}$, H. HAGINO ${ }^{1}$, Y. KAWAI ${ }^{2}$ \\ ${ }^{1}$ Department of Rehabilitation, Tottori University Hospital, Yonago City, Japan, ${ }^{2}$ Department \\ of Adaptation Physiology, Faculty of Medicine, Tottori University, Yonago City, Japan, \\ ${ }^{3}$ Department of Rehabilitation, National Hospital Organization Yonago Medical Center, Yonago \\ City, Japan
}

Received August 29, 2012

Accepted April 12, 2013

On-line July 17, 2013

\section{Summary}

This study was undertaken to investigate the effects of lower body positive pressure (LBPP) on cardiovascular responses during a 15 -min walking trial in young (22.1 \pm 0.4 years) and elderly women (67.8 \pm 1.1 years). The application of $20 \mathrm{~mm} \mathrm{Hg}$ LBPP reduced ground reaction forces by $31.2 \pm 0.5 \mathrm{kgw}$ in both groups. We hypothesized that cardiovascular responses to LBPP during walking were different between the young and elderly subjects. Applying $20 \mathrm{~mm} \mathrm{Hg}$ of LBPP increased diastolic and mean blood pressure but not systolic blood pressure in both groups. LBPPinduced reduction in heart rate (HR) occurred more quickly in the young group compared to the elderly group $(p<0.05)$. Applying LBPP also decreased double product (systolic blood pressure $x$ $\mathrm{HR}$ ) in both groups, suggesting that LBPP reduces myocardial oxygen consumption during exercise. These results suggest that heart rate responses to LBPP during exercise vary with increasing age.

\section{Key words}

Lower body positive pressure • Aging • Blood pressure • Heart rate $\bullet$ Rehabilitation

\section{Corresponding author}

Takeshi Sota, Department of Rehabilitation, Tottori University Hospital, Yonago, 683-8504, Japan. Fax: 81-859-38-6860. E-mail: tsota@med.tottori-u.ac.jp

\section{Introduction}

Lower body positive pressure (LBPP) applied on an upright human reduces apparent body weight and unloads the lower extremities (Cutuk et al. 2006), suggesting that treadmill exercise using LBPP is a new method of walking rehabilitation for patients after knee surgery (Eastlack et al. 2005). However, cardiovascular side effects of LBPP during walking should be examined in elderly patients before wide application of the method for clinical use.

Age and gender both have a significant impact on cardiovascular responses to exercise in human subjects (Martin et al. 1991, Ogawa et al. 1992). Heart rate (HR) during exercise decreases with aging over sixty years in both sexes (Ogawa et al. 1992). Systolic blood pressure (SBP) during exercise increases with aging, and the agerelated increase in SBP is greater in women than in men (Martin et al. 1991, Ogawa et al. 1992). Martin et al. (1991) suggested that calf vasodilatory capacity was reduced in old women compared with young women, but the age-dependent change was not observed in men. Proctor and coworkers (2003) investigated the difference in cardiovascular responses to leg cycle exercise using ergometer between young and old women. They reported that mean blood pressure (MBP) during exercise increased more rapidly in older women, suggesting that an increase of total vascular conductance during exercise was about $30 \%$ less in old women than in young. Overall, these reports suggest that cardiovascular responses to exercise in the elderly will vary from the young women.

Knee osteoarthritis (OA) is the most common cause of walking disability in many countries. Patients with OA have problems such as leg pain, loss of motion, 
and/or instability. The prevalence of the disease increases with advancing age (Carr et al. 2012, Menz et al. 2010, Saltzman et al. 2006). Assistive devices including canes, walkers and parallel bars are often used to walk during rehabilitation (Berman and Studenski 1998, Hesse and Werner 2003). Such rehabilitation, however, is hard for elderly patients, since these ordinary devices require muscle strength of upper extremities. Recently, an innovational method using LBPP has been introduced for rehabilitation after knee surgery (Eastlack et al. 2005, Cutuk et al. 2006).

More than 650,000 total knee replacements were done in the USA in 2008 (Carr et al. 2012). The incidence of stroke in a Japan rural area is 300-400 per 100,000 population during 1987-1991 (Morikawa et al. 2000). Many patients who have had orthopedic surgery or stroke suffer from ambulatory difficulties due to leg pain, muscle weakness, or partial paralysis in the lower extremity (Berman and Studenski 1998, Finnan et al. 2005, Kelly-Hayes et al. 2003). Applying LBPP produces a buoyant force which can reduce ground reaction forces (GRF) in an upright human (Cutuk et al. 2006), and is beneficial for gait training (Eastlack et al. 2005).

Attention should be paid, however, to an undesired side effect of LBPP that may increase blood pressure of the subject (Shi et al. 1993, Fu et al. 1998). Shi et al. (1993) reported that exposure to $20 \mathrm{~mm} \mathrm{Hg}$ LBPP produces a shift of blood volume from the legs to the thoracic compartment, an increase in central venous pressure, and an elevation of MBP in supine human subjects. Fu et al. (1998) suggested another mechanism of the increase in MBP during supine LBPP through an increase in total peripheral resistance. Nishiyasu et al.
(1998) suggested that cardiovascular responses to LBPP are largely dependent on posture. The LBPP-induced increases in MBP at rest and during ergometer exercises were greater in a supine position than that in an upright position (Nishiyasu et al. 1998, Nishiyasu et al. 2007). Nishiyasu et al. $(1998,2007)$ also reported that applying LBPP reduced HR in the upright position, but did not in the supine position. Cutuk et al. (2006) have recently reported that exposure to LBPP does not significantly affect blood pressure, but reduces HR during walking in upright human subjects whose mean age was 30 years old.

Little data is available regarding the effects of LBPP on cardiovascular responses during exercise in upright elderly subjects. The purpose of the present study was to investigate cardiovascular responses to LBPP during walking in elderly women. A blood shift toward upper body and an increase in total peripheral resistance due to LBPP might be more remarkable in elderly subjects than in young. Thus, we hypothesized that the effect of LBPP on MBP during walking is greater in elderly women than in young.

\section{Methods}

\section{Participants}

Young females $(n=20$, age: mean 22.1 years, range: 20-25 years), and post-menopausal elderly females $(n=19$, age: mean 67.8 years, range: 60-77 years) participated in this study. Included subjects did not smoke or have a history of neurological disease, orthopedic disease, cardiopulmonary disease, or metabolic disease. No subjects were taking medications

Table 1. Physical characteristics of young and elderly subjects.

\begin{tabular}{|c|c|c|}
\hline & Young $(n=20)$ & Elderly $(n=19)$ \\
\hline Age (years) & $22.1 \pm 0.4$ & $67.8 \pm 1.1^{*}$ \\
\hline Body height $(\mathrm{cm})$ & $160.0 \pm 1.1$ & $151.5 \pm 1.4^{*}$ \\
\hline Body weight (kg) & $53.6 \pm 0.9$ & $53.8 \pm 2.1$ \\
\hline$B M I\left(\mathrm{~kg} / \mathrm{m}^{2}\right)$ & $21.0 \pm 0.3$ & $23.3 \pm 0.6^{*}$ \\
\hline GRF at $20 \mathrm{~mm} \mathrm{Hg} \mathrm{LBPP} \mathrm{(kgw)}$ & $22.6 \pm 0.8$ & $22.3 \pm 1.8$ \\
\hline SBP at REST (mm Hg) & $107 \pm 4$ & $130 \pm 4^{*}$ \\
\hline DBP at REST (mm Hg) & $71 \pm 2$ & $77 \pm 2 *$ \\
\hline MBP at REST (mm Hg) & $83 \pm 2$ & $94 \pm 2 *$ \\
\hline HR at REST (beats/min) & $86.9 \pm 2.4$ & $76.4 \pm 2.5^{*}$ \\
\hline Double product at REST ( $\mathrm{mm} \mathrm{Hg \bullet beats/ \textrm {min } )}$ & $9300 \pm 460$ & $10020 \pm 470^{*}$ \\
\hline
\end{tabular}

Asterisks show statistical significance in each value between young and elderly women $(p<0.05)$. GRF, ground reaction force 


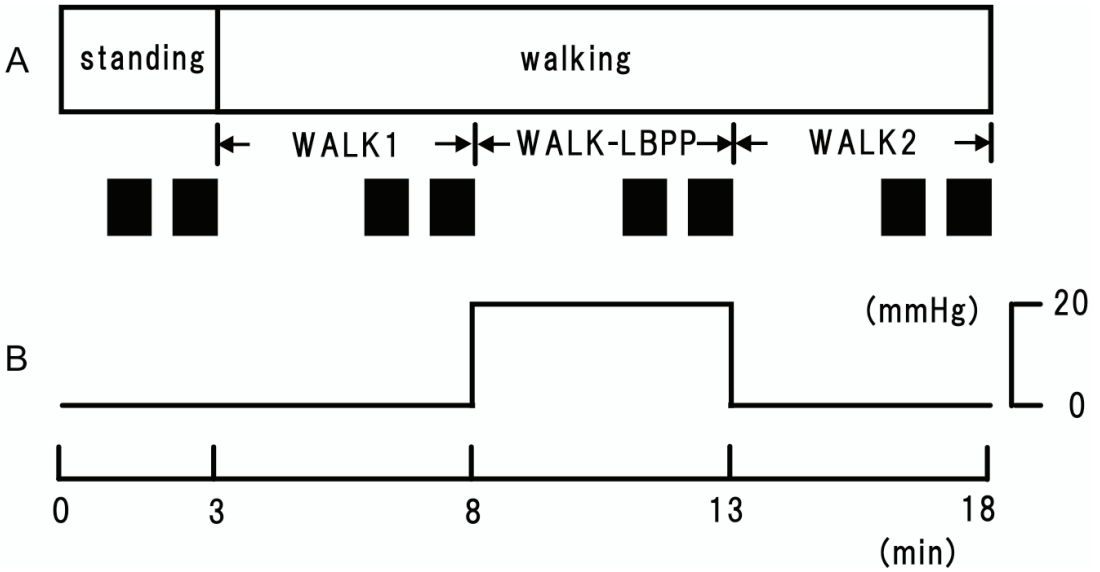

Fig. 1. Scheme of the experimental protocol. A: After $3 \mathrm{~min}$ of standing still subjects started walking for $15 \mathrm{~min}$. The square indicates the time for a measurement of blood pressure. B: 20 $\mathrm{mm} \mathrm{Hg}$ of LBPP was applied for $5 \mathrm{~min}$ during walking. having hemodynamic effects including estrogen replacement (Moreau et al. 2003). We asked all subjects about their daily physical activity by means of oral questions. No subjects participated in more than $30 \mathrm{~min}$ of moderate or severe physical activity more than 3 days per week. Six of the 20 young women and 15 of the 19 elderly women took part in $30 \mathrm{~min}$ or more of light physical activity at least one day per week for more than 6 months. Physical characteristics of both groups are presented in Table 1. Each subject signed an informed consent to participate in this study. This experimental protocol was approved by the Ethics Committee of Tottori University, Faculty of Medicine.

The room temperature was adjusted to $24-26{ }^{\circ} \mathrm{C}$, and humidity was kept constant at around $60 \%$. Subjects were instructed to avoid heavy exercise, consumption of alcohol, caffeine, or aspirin for at least 24 hours prior to the experiments. All measurements were performed between 8:30 and 12:00 in the morning. The subjects had a normal breakfast two hours before the experiments.

\section{LBPP device}

The device consists of a treadmill in an airtight chamber and a blower outside the chamber (Showa Denki Co., Ltd., Osaka, Japan) (Kawai et al. 2008). Subjects wore flexible neoprene shorts with a waist seal. They entered into the chamber through a steel ring on the top the chamber. The height of the ring was adjusted to the level of the anterior superior iliac spine of the subject. The waist seal fastened over a lip of the ring and covered the ring hole whose diameter was $60 \mathrm{~cm}$. The pressure inside the LBPP chamber could be changed from 0 to $22.5 \mathrm{~mm} \mathrm{Hg}$ above atmospheric pressure by regulating the airflow from the blower. Increased LBPP induced buoyant forces which decreased the GRF of the subject. Subjects were able to walk on the treadmill at a speed ranging of $0.2-5.0 \mathrm{~km} / \mathrm{h}$. The reductions in GRF at $20 \mathrm{~mm} \mathrm{Hg}$ LBPP in both young and elderly subjects were $31.0 \pm 0.5 \mathrm{kgw}$ and $31.5 \pm 0.8 \mathrm{kgw}$, respectively.

\section{Measurements}

Height of the subjects was measured with a height scale. A three-lead electrocardiogram (ECG) was used to monitor HR during the experiment. The ECG signal was subsequently digitized and stored at $1 \mathrm{kHz}$ with an analog-to-digital converter (Interface Corp. PCI3153; Hiroshima, Japan). The GRF of the subjects was measured with an electronic platform scale (Tanita, BWB-810; Tokyo, Japan) in the chamber without LBPP (that is body weight) or with LBPP prior to walking. SBP and diastolic blood pressure (DBP) were measured using a manual cuff applied to the right arm. The blood pressure was measured twice by the same investigator and the two values were averaged.

\section{Protocol}

On the experimental day, subjects wore the waist seal and height was measured. They went into the chamber. Then, body weight was measured with the waist seal attached to the chamber and body mass index (BMI) was calculated. GRF at $20 \mathrm{~mm} \mathrm{Hg} \mathrm{LBPP} \mathrm{were} \mathrm{measured.}$ Next, they walked on the treadmill in the chamber to practice treadmill walking. The speed of treadmill walking in the practice section was increased from 0.2 to $3.0 \mathrm{~km} / \mathrm{h}$ in a stepwise manner. All subjects soon became familiar with walking without holding the handrails. After completion of the walking practice trial, they rested $5 \mathrm{~min}$ in the sitting position outside the chamber. They then started an exercise protocol as shown in Figure 1. The subjects stood still for $3 \mathrm{~min}$ (REST) and then walked on the treadmill at a speed of $3 \mathrm{~km} / \mathrm{h}$ for $15 \mathrm{~min}$. They walked without LBPP (WALK1) for the first 5-min 
period, walked with $20 \mathrm{~mm} \mathrm{Hg} \mathrm{LBPP} \mathrm{(WALK-LBPP)} \mathrm{for}$ the next 5-min period, and walked without LBPP (WALK2) for the third 5-min period. Blood pressure was measured during the last 2 min of each section.

\section{Data analysis}

HR was calculated from the RR interval by offline analysis using MATLAB (MathWorks; Natick, MA, USA). HR was averaged every $60 \mathrm{~s}$. Maximal heart rate $\left(\mathrm{HR}_{\max }\right)$ was estimated using the age-predicted equation in healthy women (Tanaka et al. 2001). A percentage of $\mathrm{HR}_{\max }\left(\% \mathrm{HR}_{\max }\right)$ was used to evaluate exercise intensity. MBP was calculated as DBP plus one third of the pulse pressure. The product of HR and SBP, the double product, was used as an index of myocardial oxygen consumption (Nelson et al. 1974). All data were presented as mean $\pm \mathrm{SE}$ (standard errors of the mean).

SPSS version 11.0 was used for statistical analysis (IBM Japan Ltd; Tokyo, Japan). A mixed-design analysis of variance (ANOVA) was used to compare mean values of SBP, DBP, MBP, HR and double product between WALK1, WALK-LBPP, and WALK2. Two factor factorial ANOVA was used to compare response time of HR to LBPP between young and elderly subjects. Tukey-Kramer's test was used as a post-hoc analysis. In order to examine the effect of walking itself on the cardiovascular parameters, a paired t-test was used to compare the data between REST and WALK1. A $p$ value of less than 0.05 was considered significant.

\section{Results}

\section{Physical characteristics of the subjects}

Mean body weight did not vary between the young and elderly groups (Table 1). The mean height of young subjects was higher than that of elderly subjects. The average BMI of the young group was $21.0 \pm 0.3 \mathrm{~kg} / \mathrm{m}^{2}$ and that of the elderly group was $23.3 \pm 0.6 \mathrm{~kg} / \mathrm{m}^{2}$. Applying $20 \mathrm{~mm} \mathrm{Hg}$ of LBPP reduced the GRF of the subjects to $22.6 \pm 0.8 \mathrm{kgw}$ and $22.3 \pm 1.8 \mathrm{kgw}$ in the young and elderly groups, respectively. SBP, DBP and MBP at REST of the elderly subjects were higher than those of the young subjects, respectively. HR at REST of the old subjects was lower than that of the young subjects. Double product at REST of the old subjects was significantly greater than that of the young subjects.

\section{Effects of LBPP on blood pressure during walking}

Figure 2 shows changes in blood pressure during walking on the treadmill in the LBPP chamber. There was no interaction between the group (elderly and young subjects) and time factors (WALK1, WALK-LBPP, and WALK2). In elderly subjects, SBP at REST was $130 \pm 4$ $\mathrm{mm} \mathrm{Hg}$ (Table 1) and increased to $143 \pm 4 \mathrm{~mm} \mathrm{Hg}$ during WALK1 ( $p=0.001$ ). Applying LBPP did not affect SBP significantly in elderly subjects. The SBP in the young group was $107 \pm 4 \mathrm{~mm} \mathrm{Hg}$ at REST (Table 1) and did not change during WALK1. Applying LBPP did not increase SBP significantly also in young subjects.

The DBP during WALK1 in elderly subjects was $74 \pm 2 \mathrm{~mm} \mathrm{Hg}$, increased at the end of WALK-LBPP ( $\%$ increase of DBP: $3.3 \pm 1.6 \%, p=0.02$ ), and then decreased after the WALK2 period (\% decrease of DBP: $4.7 \pm 1.8 \%, \mathrm{p}=0.0002)$. The DBP during WALK1 in young group was $63 \pm 2 \mathrm{~mm} \mathrm{Hg}$, increased at the end of WALK-LBPP (\% increase of DBP: $3.7 \pm 1.4 \%, \mathrm{p}=0.02$ ) and decreased at the end of WALK2 $(\%$ decrease of DBP: $5.6 \pm 1.4 \%, \mathrm{p}=0.0002$ ).

The MBP during WALK1 in elderly subjects was $97 \pm 2 \mathrm{~mm} \mathrm{Hg}$, increased at the end of WALK-LBPP (\% increase of MBP: $2.3 \pm 1.2 \%, \mathrm{p}=0.04$ ) and decreased during WALK2 (\% decrease of MBP: $3.6 \pm 1.4 \%$, $\mathrm{p}=0.002$ ). The MBP during WALK1 in the young subjects was $78 \pm 2 \mathrm{~mm} \mathrm{Hg}$, increased at the end of WALK-LBPP (\% increase of MBP: $2.0 \pm 0.9 \%, \mathrm{p}=0.04$ ) and decreased during WALK2 (\% decrease of MBP: $3.4 \pm 1.1 \%, \mathrm{p}=0.002$ ).

\section{Effects of LBPP on changes in HR during walking}

Figure 3A shows effect of LBPP on HR during walking. In the elderly group, the mean HR was $76.4 \pm 2.5$ beats $/ \min \left(\% \mathrm{HR}_{\max }: 46.9 \pm 1.2 \%\right)$ at REST and increased to $92.4 \pm 2.6$ beats/min $\left(\% \mathrm{HR}_{\max }: 56.6 \pm 1.6 \%\right)$ at the end of WALK1. HR decreased gradually and reached a significantly lower value $4 \mathrm{~min}$ after the onset of LBPP application. This decreased HR returned slowly to the pre-LBPP level in the elderly group.

The mean HR at REST in young subjects was $86.9 \pm 2.4$ beats $/ \min \left(\% \mathrm{HR}_{\max }: 45.1 \pm 1.5 \%\right)$ and increased to $97.9 \pm 2.5$ beats/min $\left(\% \mathrm{HR}_{\max }: 50.7 \pm 1.3 \%\right)$ at the end of WALK1. Exposure to LBPP significantly decreased HR within a minute after its onset in young subjects. The decreased HR recovered quickly within a minute after cessation of LBPP to the level of WALK1.

In Figure 3B, we compared the response time of HR to LBPP (elapsed time for $2 \%, 4 \%$, and $6 \%$ reduction from the baseline value) between young and elderly subjects. Response time for $2 \%$ reduction in the 
elderly group was not different from that in the young group, but the response times for $4 \%$ and $6 \%$ reduction in the elderly subjects were significantly longer than those in the young subjects $(\mathrm{p}=0.048$ and $\mathrm{p}=0.0005$, respectively). Response times for $4 \%$ and $6 \%$ increase during the recovery after WALK-LBPP were also longer in the elderly group than in the young as shown in Figure $3 \mathrm{C}(\mathrm{p}=0.03$ and $\mathrm{p}=0.0002$, respectively).

\section{elderly}
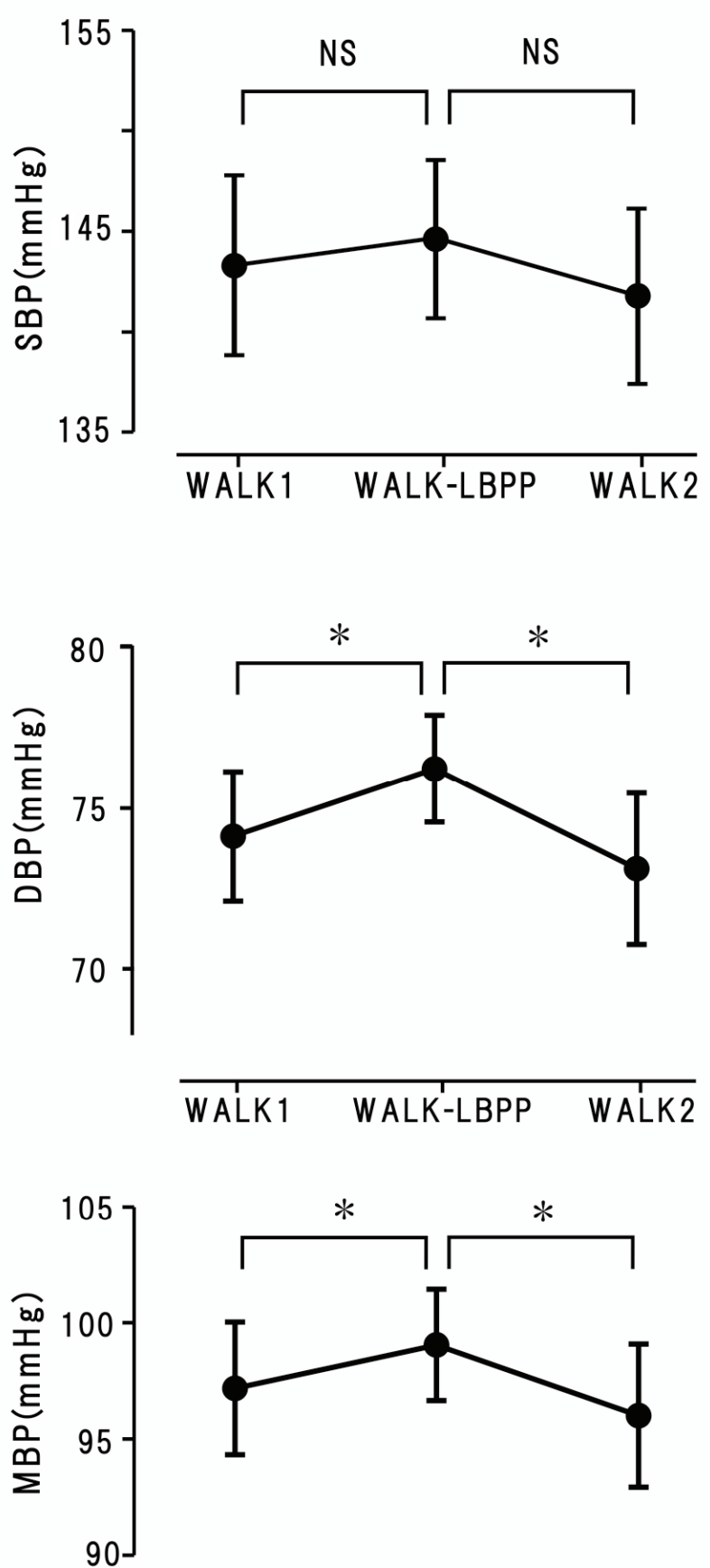

WALK1 WALK'LBPP $\quad$ WALK2 young
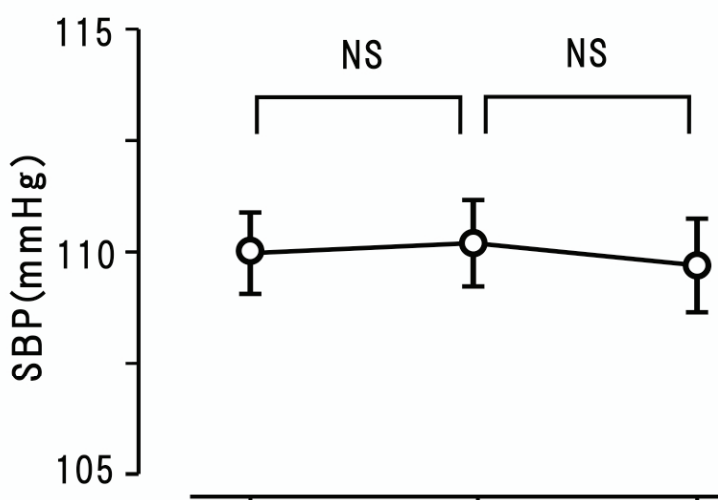
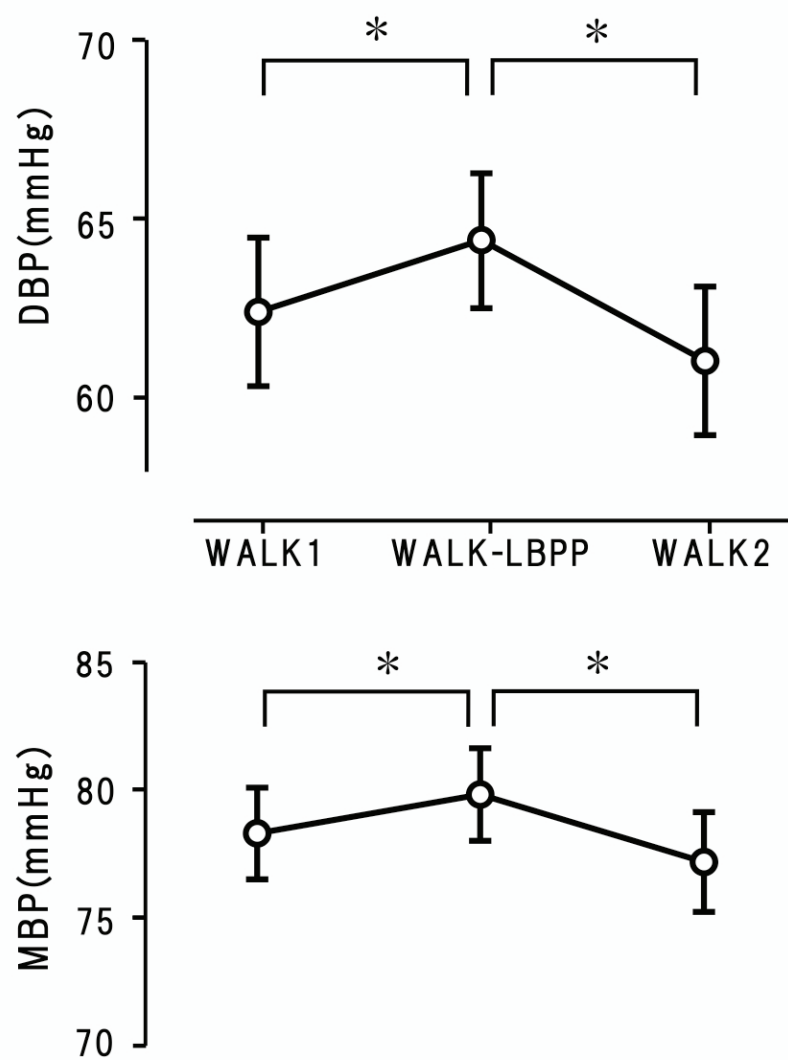

WALK1 WALK-LBPP $\quad$ WALK2

Fig. 2. Effect of LBPP on blood pressure during walking in elderly (left panels) and young women (right panels). Panels show SBP, DBP, and MBP from the top to the bottom.* $p<0.05$, significant differences between the values during WALK1, WALK-LBPP and WALK2 (Tukey-Kramer's test after mixed-design ANOVA) 
A

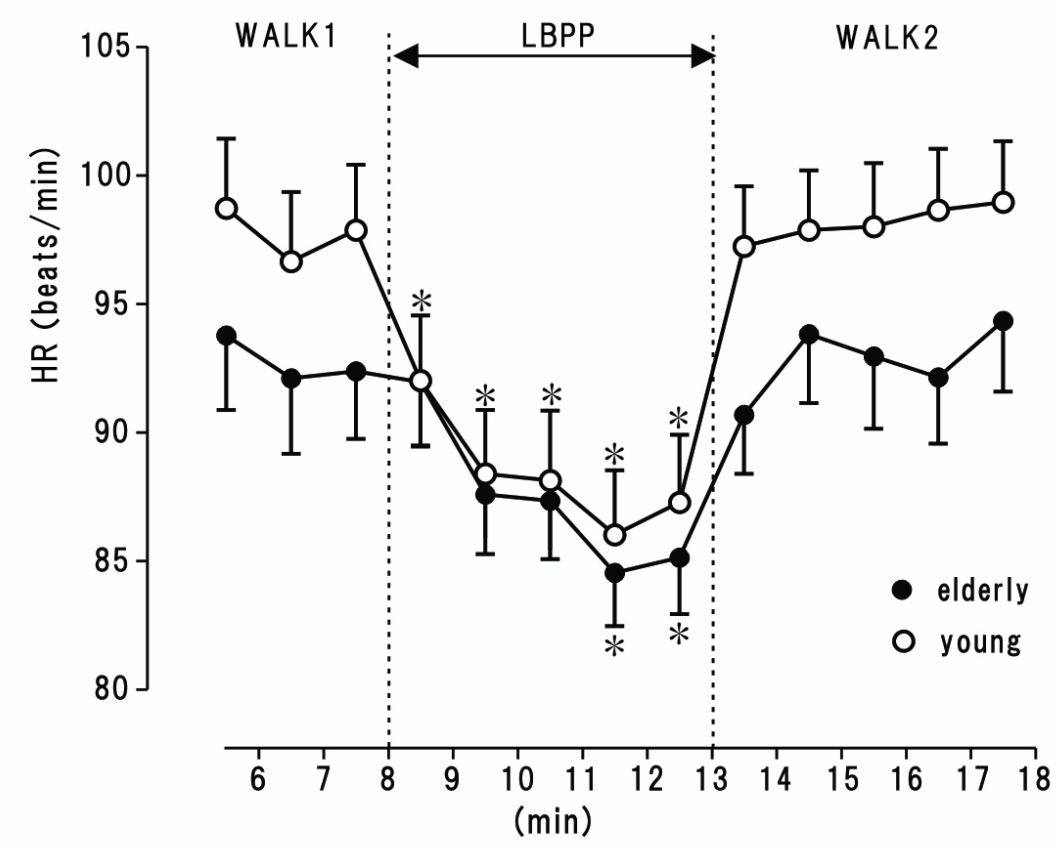

B

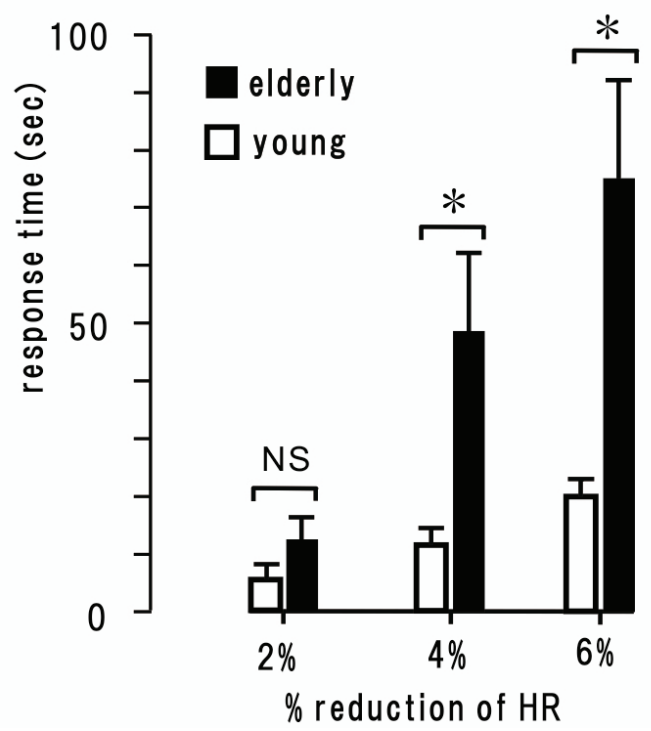

C

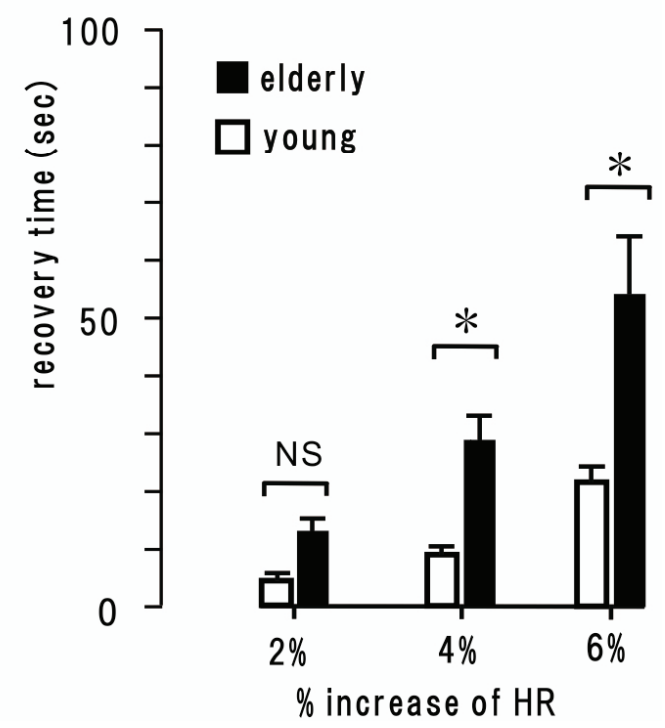

Fig. 3. A: Effect of LBPP on HR during walking in young and elderly women. $* p<0.05$ compared to mean HR during the 1 min after onset of walking (Tukey-Kramer's test after mixed-design ANOVA); B: Effect of LBPP on times from onset of LBPP to $2 \%, 4 \%$, and $6 \%$ reductions in HR during walking in young and elderly women. These reductions in HR compared to mean HR during the 1 min before onset of applying LBPP. * $p<0.05$, significant differences between the young and elderly women (Tukey-Kramer's test after 2 way factorial ANOVA); C: Recovery time from offset of LBPP to $2 \%, 4 \%$, and $6 \%$ increases in HR during walking in young and elderly women. The increases in HR compared to mean HR during the 1 min before offset of LBPP. $* p<0.05$, significant differences between the young and elderly women (Tukey-Kramer's test after 2-way factorial ANOVA)

\section{Effects of LBPP on double product during walking}

Figure 4 shows changes in double product during walking. There was no interaction between the group and time factors. In the elderly group, the double product at REST was $10020 \pm 470 \mathrm{~mm} \mathrm{Hg} \bullet$ beats/min (Table 1) and increased significantly at the end of WALK1 $(p=0.00000002)$. The double product decreased significantly during WALK-LBPP $(\mathrm{p}=0.00008)$, and then increased again by the end of WALK2 $(\mathrm{p}=0.0006)$. The double product in the young group was $9300 \pm 460 \mathrm{~mm}$ Hg•beats/min at REST (Table 1) and increased significantly at the end of WALK1 $(\mathrm{p}=0.0000002)$. The double product decreased during WALK-LBPP $(\mathrm{p}=0.000008)$ and increased during WALK2 $(\mathrm{p}=0.000006)$. 


\section{- elderly \\ O young}

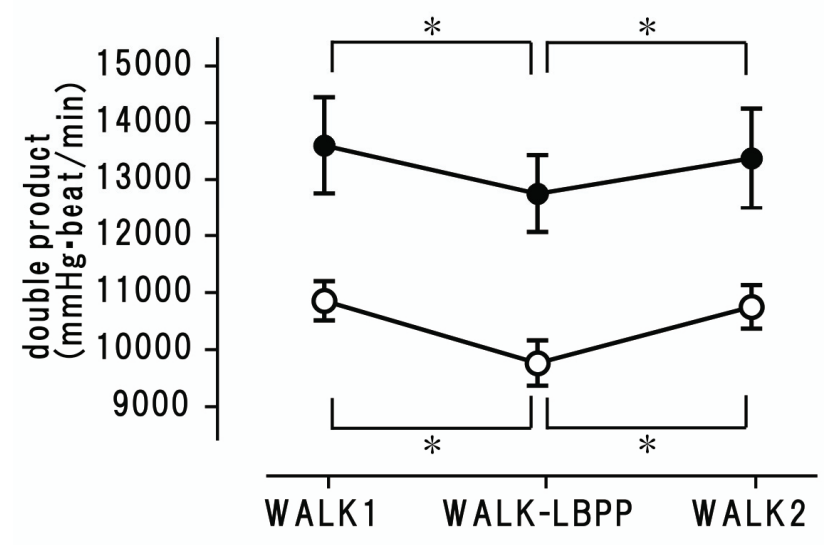

Fig. 4. Effect of LBPP on double product (SBP $x$ HR) during walking in young and elderly women. $* p<0.05$, significant differences between the values during WALK1, WALK-LBPP and WALK2 (Tukey-Kramer's test after mixed-design ANOVA)

\section{Discussion}

The major findings of this study are: 1) Applying LBPP increased the DBP and MBP during walking in the elderly and young women, 2) The LBPPinduced reduction in HR occurred more quickly in young subjects than in elderly ones, and 3) Exposure to LBPP reduced double product during walking in both groups.

Various studies have reported the effects of applying LBPP on the blood pressure at rest and/or during exercise. The cardiovascular responses to LBPP seem to vary depending on differences in the posture of the subjects. Shi et al. (1993) showed that exposure to $40 \mathrm{~mm} \mathrm{Hg} \mathrm{LBPP} \mathrm{increased} \mathrm{the} \mathrm{MBP} \mathrm{at} \mathrm{rest} \mathrm{from} 86$ to $95 \mathrm{~mm} \mathrm{Hg}$ in the supine position. The MBP during ergometer exercise in the supine position is also increased by applying $25-75 \mathrm{~mm} \mathrm{Hg}$ of LBPP (Nishiyasu et al. 1998). The elevation of MBP produced by LBPP is partly attributable to translocation of blood volume from the leg to the thorax which increases cardiac preload and stroke volume (Shi et al. 1993). Other mechanisms for the elevation of MBP include muscle mechanoreflex and metaboreflex. Application of LBPP causes an increase of intramuscular pressure which in turn activates a muscle mechanoreflex (Fu et al. 1998, Stebbins et al. 1988, Williamson et al. 1994), resulting in an elevation of MBP. Furthermore, exposure of the legs to LBPP during exercise limits blood supply to the acting muscles (Macias et al. 2012), accumulates metabolites in the tissue, and elicits a pressor metaboreflex (Williamson et al. 1994, Eiken and Bjurstedt 1987).

On the other hand, blood pressure does not change significantly with LBPP in subjects in a standing position (Eastlack et al. 2005, Cutuk et al. 2006, Kawai et al. 2008), or LBPP-induced increase in blood pressure is greater in a supine position than in a standing position (Nishiyasu et al. 1998, Nishiyasu et al. 2007). The present data in young subjects are consistent with these previous results in that the increase in MBP due to LBPP is minimal in standing subjects (Fig. 2). The posturedependent difference in the effect of LBPP on HR and blood pressure has not been fully understood. Nishiyasu et al. (2007) demonstrated an increase in stroke volume during the application of $25-50 \mathrm{~mm} \mathrm{Hg}$ of LBPP in an upright position. However, HR is decreased at the same time, which may cancel an increase in cardiac output otherwise induced by the increased stroke volume. Another possibility for the lack of blood pressure elevation in upright LBPP may be an effect of hydrostatic pressure which prevents an increase in venous return from the legs in upright posture. Furthermore, relatively lower level of LBPP was applied in the present study, which may also explain our results. Although the mechanism is unclear, the smaller pressor response to standing LBPP in contrast to LBPP in supine position supports the use of this device in rehabilitation.

It has been shown that age and gender have a significant impact on cardiovascular responses to exercise. In this study, both elderly and young subjects walked at the same speed. As a result, $\% \mathrm{HR}_{\max }$ at the end of WALK1 in the elderly subjects $(56.6 \%)$ was greater than that in the young subjects $(50.7 \%)$. These exercise intensities based on the $\% \mathrm{HR}_{\max }$ in both subjects, however, were classified as light exercise according to the previous report (Garber et al. 2011), suggesting that both young and elderly subjects did not reach an anaerobic threshold in this study. In general, the elevation of blood pressure and systemic vascular resistance during submaximal exercise in elderly women is larger than that in elderly men or young women (Fleg et al. 1995, Ogawa et al. 1992). This variation in the MBP response between the young and elderly groups seems to be attributable to age-related changes in cardiovascular function (Fleg et al. 1995, Ogawa et al. 1992, Kawai and Ohhashi 1990, Muller-Delp et al. 2002, Proctor et al. 2003). Relaxant responses of isolated rat vascular smooth muscles to vasodilator agents are reduced with increasing age (Kawai and Ohhashi 1990). Responses of rat skeletal muscle arterioles to acetylcholine are attenuated in older 
animals, suggesting that aging impairs endotheliumdependent vasodilation (Muller-Delp et al. 2002). Impairment of vasodilator responses during exercise has been described also in human subjects (Fleg et al. 1995, Martin et al. 1991, Ogawa et al. 1992, Proctor et al. 2003).

Applying LBPP reduced HR during walking exercise in both young and elderly women in this study. The results of the present study are consistent with previous observations (Eastlack et al. 2005, Cutuk et al. 2006, Hoffman and Donaghe 2011). The LBPP effect on HR depends on the posture of the subjects with a greater drop of HR in the upright position, but no change in the supine position (Nishiyasu et al. 1998, Nishiyasu et al. 2007). A possible mechanism to describe the reduction in $\mathrm{HR}$ is attributed to a decrease in walking energy cost by body weight unloading (Grabowski et al. 2005, Ruckstuhl et al. 2009). A previous study showed that unloading using a device of harness type, without positive pressure on the lower body, also reduced HR during treadmill walking (Ruckstuhl et al. 2009). Another mechanism seems to be related to baroreceptor reflex induced by translocation of blood volume from the lower body to the thorax due to application of LBPP. Interestingly, Nishiyasu et al. (2007) demonstrated that the LBPP-induced increase in stroke volume or cardiac output was greater in the upright position than in the supine position. They discussed that higher hydrostatic pressures caused more blood pooling while in the upright position compared to the supine position. If so, larger amount of blood may shift toward the upper body during upright LBPP. Thus, arterial baroreceptors might be activated more strongly by the application of LBPP in the upright posture than in the supine position.

The present study demonstrated that the LBPPinduced decrease of HR during walking occurred more slowly in elderly subjects compared to young subjects. This difference is not attributable to body weight unloading since the application of LBPP reduced the GRF of the subjects by the same extent in both groups (Table 1). One possible explanation for this is ageassociated impairment of baroreflex sensitivity (Laitinen et al. 1998), which may delay the response of HR to LBPP in elderly subjects.

On the other hand, our study showed that the LBPP-induced increase of MBP during walking in elderly group was not different from that in young group (Fig. 2). If aging decreases baroreflex sensitivity as mentioned above, the LBPP-induced increase of MBP in elderly group should be larger than that in young group.

\section{Future research}

Our results suggest that the response of baroreflex in young women to LBPP might be quicker than that in elderly. However, we could not detect an agedependent change in blood pressure response to LBPP. One possible reason for this is that the blood pressure was measured after $3 \mathrm{~min}$ from the onset of LBPP. If we were able to measure the blood pressure at earlier phase, we might find a difference in the LBPP-induced response between young and elderly subjects. Continuous monitoring of blood pressure will be needed in future study.

Another issue that we should concern is the difference in relative workload that the young $(50.7 \%$ of $\left.\% \mathrm{HR}_{\max }\right)$ and elderly $\left(56.6 \%\right.$ of $\left.\% \mathrm{HR}_{\max }\right)$ subjects exercised. If an equal level of relative workload was employed, a different result may have been obtained.

Martin et al. (1991) suggested that estrogen modulates vascular tone. In the present study, postmenopausal women were used as the elderly subjects, and menstruation was not controlled in young subjects. The effects of menstruation cycle and/or hormone replacement therapy will be interesting issues to concern in future studies. There have been no reports in which cardiovascular responses to LBPP were investigated in elderly male subjects. In order to address the genderrelated difference more precisely, we need to examine elderly male subjects in a future study.

\section{Implications of the study}

The present results suggest that treadmill walking in a LBPP chamber is useful for a safe and comfortable rehabilitation for patients with weightbearing restrictions, muscle atrophy, or partial paralysis. The application of LBPP reduced HR and double product $(\mathrm{HR} \times \mathrm{SBP})$ during walking in both young and elderly women (Figs 3 and 4). Since double product correlates closely with myocardial oxygen consumption (Nelson et al. 1974), our results suggest that the application of LBPP during exercise reduces myocardial oxygen consumption. For clinical use, however, attention should be paid to a small elevation of MBP (Fig. 2). The LBPP-induced elevation of MBP is proportional to the level of the LBPP applied (Nishiyasu et al. 1998, Nishiyasu et al. 2007). Therefore, the lower the LBPP, the safer the rehabilitation. The LBPP-produced buoyant force is determined by the product of area of the waist seal and 
pressure difference between the inside and outside of the chamber, i.e., LBPP. In order to achieve adequate buoyant forces with a minimum LBPP, the area of the waist seal should be large. A reinforcing synthetic rubber with high elasticity was pasted on the inside of the waist seal to prevent too much ballooning of the large waist seal when LBPP was applied. With our device having a large waist seal area, a reduction of GRF by $30 \mathrm{kgw}$ was achieved by $20 \mathrm{~mm} \mathrm{Hg}$ of LBPP which caused only a 2-3 $\mathrm{mm} \mathrm{Hg}$ elevation of MBP even in elderly subjects.

In conclusion, applying LBPP during treadmill walking slightly increased MBP in both groups, but there was no significant difference in the MBP response between the groups. LBPP reduced HR and double product in both young and elderly groups. The LBPPinduced change in HR occurred more slowly in elderly subjects compared to young subjects. These results suggest that heart rate responses to LBPP during exercise vary with increasing age.

\section{Conflict of Interest}

There is no conflict of interest.

\section{Acknowledgements}

A part of this work was carried out in collaboration with Showa Denki Co., Ltd.

\section{References}

BERMAN A, STUDENSKI S: Musculoskeletal rehabilitation. Clin Geriatr Med 14: 641-659, 1998.

CARR AJ, ROBERTSSON O, GRAVES S, PRICE AJ, ARDEN NK, JUDGE A, BEARD DJ: Knee replacement. Lancet 379: 1331-1340, 2012.

CUTUK A, GROPPO ER, QUIGLEY EJ, WHITE KW, PEDOWITZ RA, HARGENS AR: Ambulation in simulated fractional gravity using lower body positive pressure: cardiovascular safety and gait analyses. J Appl Physiol 101: 771-777, 2006.

EASTLACK RK, HARGENS AR, GROPPO ER, STEINBACH GC, WHITE KK, PEDOWITZ RA: Lower body positive-pressure exercise after knee surgery. Clin Orthop Relat Res 431: 213-219, 2005.

EIKEN O, BJURSTEDT H: Dynamic exercise in man as influenced by experimental restriction of blood flow in the working muscles. Acta Physiol Scand 131: 339-345, 1987.

FINNAN R, FUNK L, PINZUR MS, RABIN S, LOMASNEY L, JUKENELIS D: Health related quality of life in patients with supination-external rotation stage IV ankle fractures. Foot Ankle Int 26: 1038-1041, 2005.

FLEG JL, O'CONNOR F, GERSTENBLITH G, BECKER LC, CLULOW J, SCHULMAN SP, LAKATTA EG: Impact of age on the cardiovascular response to dynamic upright exercise in healthy men and women. $J$ Appl Physiol 78: 890-900, 1995.

FU Q, SUGIYAMA Y, KAMIYA A, SHAMSUZZAMAN AS, MANO T: Responses of muscle sympathetic nerve activity to lower body positive pressure. Am J Physiol 275: H1254-H1259, 1998.

FU Q, IWASE S, NIIMI Y, KAMIYA A, KAWANOKUCHI J, CUI J, MANO T, SUZUMURA A: Effects of lower body positive pressure on muscle sympathetic nerve activity response to head-up tilt. Am J Physiol 281: R778R785, 2001.

GARBER CE, BLISSMER B, DESCHENES MR, FRANKLIN BA, LAMONTE MJ, LEE IM, NIEMAN DC, SWAIN DP: American College of Sports Medicine position stand. Quantity and quality of exercise for developing and maintaining cardiorespiratory, musculoskeletal, and neuromotor fitness in apparently healthy adults: guidance for prescribing exercise. Med Sci Sports Exerc 43: 1334-1359, 2011.

GRABOWSKI A, FARLEY CT, KRAM R: Independent metabolic costs of supporting body weight and accelerating body mass during walking. J Appl Physiol 98: 579-583, 2005.

HESSE S, WERNER C: Partial body weight supported treadmill training for gait recovery following stroke. Adv Neurol 92: 423-428, 2003.

HOFFMAN MD, DONAGHE HE: Physiological responses to body weight-supported treadmill exercise in healthy adults. Arch Phys Med Rehabil 92: 960-966, 2011.

KAWAI Y, OHHASHI T: Age-related changes in relaxant response of vascular smooth muscles to atrial natriuretic peptide. J Pharmacol Exp Ther 252: 1234-1239, 1990. 
KAWAI Y, MATSUO S, OHNISHI H, TAGAWA K, HAYASHI T: Cardiovascular responses to lower body positive pressure. Adaptation Biol Med 5: 29-36, 2008.

KELLY-HAYES M, BEISER A, KASE CS, SCARAMUCCI A, D'AGOSTINO RB, WOLF PA: The influence of gender and age on disability following ischemic stroke: the Framingham study. J Stroke Cerebrovasc Dis 12: 119-126, 2003.

LAITINEN T, HARTIKAINEN J, VANNINEN E, NISKANEN L, GEELEN G, LANSIMIES E: Age and gender dependency of baroreflex sensitivity in healthy subjects. J Appl Physiol 84: 576-583, 1998.

MACIAS BR, D'LIMA DD, CUTUK A, PATIL S, STEKLOV N, NEUSCHWANDER TB, MEUCHE S, COLWELL CW, HARGENS AR: Leg intramuscular pressures and in vivo knee forces during lower body positive and negative pressure treadmill exercise. J Appl Physiol 113: 31-38, 2012.

MARTIN WH, OGAWA T, KOHRT WM, MALLEY MT, KORTE E, KIEFFER PS, SCHECHTMAN KB: Effects of aging, gender, and physical training on peripheral vascular function. Circulation 84: 654-664, 1991.

MENZ HB, JORDAN KP, RODDY E, CROFT PR: Musculoskeletal foot problems in primary care: what influences older people to consult? Rheumatology (Oxford) 49: 2109-2116, 2010.

MOREAU KL, DONATO AJ, TANAKA H, JONES PP, GATES PE, SEALS DR: Basal leg blood flow in healthy women is related to age and hormone replacement therapy status. $J$ Physiol 547: 309-316, 2003.

MORIKAWA Y, NAKAGAWA H, NARUSE Y, NISHIJO M, MIURA K, TABATA M, HIROKAWA W, KAGAMIMORI S, HONDA M, YOSHITA K, HAYASHI K: Trends in stroke incidence and acute case fatality in a Japanese rural area: the Oyabe study. Stroke 31: 1583-1587, 2000.

MULLER-DELP JM, SPIER SA, RAMSEY MW, DELP MD: Aging impairs endothelium-dependent vasodilation in rat skeletal muscle arterioles. Am J Physiol 283: H1662-H1672, 2002.

NELSON RR, GOBEL FL, JORGENSEN CR, WANG K, WANG Y, TAYLOR HL: Hemodynamic predictors of myocardial oxygen consumption during static and dynamic exercise. Circulation 50: 1179-1189, 1974.

NISHIYASU T, NAGASHIMA K, NADEL ER, MACK GW: Effects of posture on cardiovascular responses to lower body positive pressure at rest and during dynamic exercise. J Appl Physiol 85: 160-167, 1998.

NISHIYASU T, HAYASHIDA S, KITANO A, NAGASHIMA K, ICHINOSE M: Effects of posture on peripheral vascular responses to lower body positive pressure. Am J Physiol 293: H670-H676, 2007.

OGAWA T, SPINA RJ, MARTIN WH, KOHRT WM, SCHECHTMAN KB, HOLLOSZY JO, EHSANI AA: Effects of aging, sex, and physical training on cardiovascular responses to exercise. Circulation 86: 494-503, 1992.

PROCTOR DN, KOCH DW, NEWCOMER SC, LE KU, LEUENBERGER UA: Impaired leg vasodilation during dynamic exercise in healthy older women. J Appl Physiol 95: 1963-1970, 2003.

RENEMAN RS, SLAAF DW, LINDBOM L, TANGELDER GJ, ARFORS KE: Muscle blood flow disturbances produced by simultaneously elevated venous and total muscle tissue pressure. Microvasc Res 20: 307-318, 1980.

RUCKSTUHL H, KHO J, WEED M, WILKINSON MW, HARGENS AR: Comparing two devices of suspended treadmill walking by varying body unloading and Froude number. Gait Posture 30: 446-451, 2009.

SALTZMAN CL, ZIMMERMAN MB, O'ROURKE M, BROWN TD, BUCKWALTER JA, JOHNSTON R: Impact of comorbidities on the measurement of health in patients with ankle osteoarthritis. J Bone Joint Surg Am 88: 2366-2372, 2006.

SHI X, CRANDALL CG, RAVEN PB: Hemodynamic responses to graded lower body positive pressure. Am J Physiol 265: H69-H73, 1993.

STEBBINS CL, BROWN B, LEVIN D, LONGHURST JC: Reflex effect of skeletal muscle mechanoreceptor stimulation on the cardiovascular system. J Appl Physiol 65: 1539-1547, 1988.

TANAKA H, MONAHAN KD, SEALS DR: Age-predicted maximal heart rate revisited. J Am Coll Cardiol 37: 153$156,2001$.

WILLIAMSON JW, MITCHELL JH, OLESEN HL, RAVEN PB, SECHER NH: Reflex increase in blood pressure induced by leg compression in man. J Physiol 475: 351-357, 1994. 